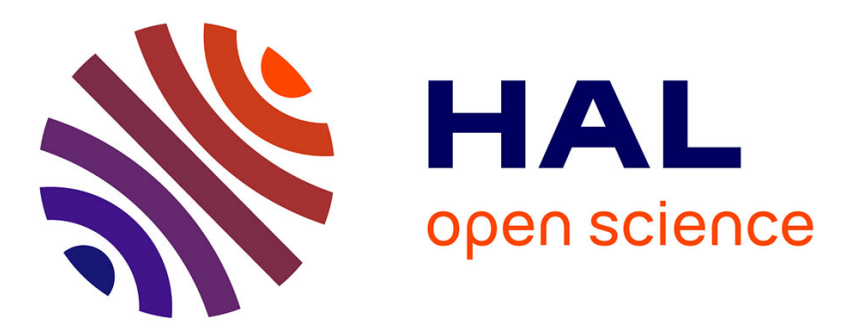

\title{
Should renin-angiotensin-aldosterone system inhibition enablement be a therapeutic target in CKD patients?
}

Patrick Rossignol, Rajiv Agarwal

\section{To cite this version:}

Patrick Rossignol, Rajiv Agarwal. Should renin-angiotensin-aldosterone system inhibition enablement be a therapeutic target in CKD patients?. Nephrology Dialysis Transplantation, 2021, 36 (10), pp.1771-1772. 10.1093/ndt/gfab061 . hal-03274455

\section{HAL Id: hal-03274455 \\ https://hal.univ-lorraine.fr/hal-03274455}

Submitted on 30 Jun 2021

HAL is a multi-disciplinary open access archive for the deposit and dissemination of scientific research documents, whether they are published or not. The documents may come from teaching and research institutions in France or abroad, or from public or private research centers.
L'archive ouverte pluridisciplinaire HAL, est destinée au dépôt et à la diffusion de documents scientifiques de niveau recherche, publiés ou non, émanant des établissements d'enseignement et de recherche français ou étrangers, des laboratoires publics ou privés. 


\section{Should renin angiotensin aldosterone system inhibition enablement be a therapeutic target in CKD patients?}

Patrick Rossignol $^{1}$, Rajiv Agarwal ${ }^{2}$

1. Université de Lorraine, INSERM, Centre d'Investigations Cliniques-Plurithématique 14-33, and INSERM U1116, CHRU de Nancy, F-CRIN INI-CRCT (Cardiovascular and Renal Clinical Trialists), France

2. Department of Medicine, Division of Nephrology, Indiana University School of Medicine, Indianapolis, IN, USA

Renin angiotensin aldosterone system (RAAS) inhibitor use is of paramount importance in patients with diabetes, hypertension, heart failure and reduced ejection fraction (HFrEF- the latter frequently treated with a combination of angiotensin converting enzyme inhibitors/angiotensin receptor blockers (ACE/ARB) and mineralocorticoid receptor antagonists (MRA)), and chronic kidney disease (CKD) with albuminuria. Their use is strongly recommended by evidence-based cardiology and nephrology guidelines $^{1}$; physician adherence to guideline-directed medical therapy in HFrEF associates with improved outcomes.

In the United States, a recent survey among 38,885 adult National Health and Nutrition Examination Survey participants with eGFR $<60 \mathrm{ml} / \mathrm{min}$ per $1.73 \mathrm{~m}^{2}$ or urinary albumin-to-creatinine ratio $\geq 30$ $\mathrm{mg} / \mathrm{g}$ showed that although the use of ACE/ARB increased between 1999 and 2014, it appeared to plateau after 2003 , with approximately only $40 \%$ of the CKD population using an ACE/ARB ${ }^{2}$. Of note, within the Chronic Kidney Disease Outcomes and Practice Patterns Study (CKDopps) there are substantial variations among countries in RAASi prescription; RAASi prescription was found less common (52\%) in the United States when compared to Germany (80\%), France $(77 \%)$ and Brazil $(66 \%)^{3}$. These differences were particularly pronounced in patients with later stages of CKD.

In this issue of NDT, Walther et al. ${ }^{4}$ in a cohort of 1,371,075 older US veterans with CKD identified 141,252 patients who were given a new prescription of ACEI/ARB; (17.4\% had congestive heart failure). They reported that ACEI/ARB discontinuation was associated with an approximately two-fold increased risk of death and about 1.5 fold increase in the risk of end-stage kidney disease. This analysis robustly expands the thus far sparse knowledge regarding the association of ACEI/ARB discontinuation with long-term outcomes in CKD, also elegantly reviewed in their report. Aside from the observational design precluding the ascertainment of causality, a limitation acknowledged by the authors, was the inability to determine within the framework of this database the exact circumstances of ACEI/ARB discontinuation. One reason they rightly propose is frailty or limited projected life expectancy.

Others include worsening of renal function, whether it be with or without acute kidney injury, hypotension and hyperkalaemia. Similar results are reported by others. For example, among 205,108 US patients including more than 20,000 heart failure patients, nearly $60 \%$ who discontinued RAASi after an hyperkalaemic episode experienced an adverse outcome or mortality. Heart failure patients on submaximum dose, or who discontinued RAASi died twice as frequently as patients on maximum dose. Over $50 \%$ of the 43,388 patients with CKD stages 3 to 4 who discontinued RAAS inhibitors 
experienced an adverse outcome or died ${ }^{5}$. In a large (9,222 outpatients, 20.3\% with CKD) European chronic heart failure patient database, mediation analyses showed that hyperkalaemia $\geq 5.5 \mathrm{mmol} / \mathrm{L}$ was associated with all-cause mortality and cardiovascular mortality by mediating discontinuation of ACEi (marginally significant for all-cause death: $P=0.059$ ), ARB (not statistically significant), and MRA $\left(P=0.007\right.$ for all-cause death; $P=0.080$ for cardiovascular death) ${ }^{6}$, suggesting that hyperkalaemia could represent a risk factor for RAASi discontinuation and the discontinuation of MRA in particular due to hyperkalaemia associates with worse outcomes,

Interestingly, a patient-level simulation model was designed to fully characterise the natural history of CKD over a lifetime horizon, and predict the associations between serum potassium levels, RAASi use and long-term outcomes based on published literature. This analysis supported the notion that maintaining normokalaemia (using guideline-recommended treatments) is a valuable strategy to enable optimal RAASi therapy and improve long-term health economic outcomes in CKD patients ${ }^{7}$.

Whether the vicious circle surrounding hyperkalaemia, which is an inherent risk factor for RAASi under-use, under-dosing and discontinuation, may be overcome by the availability of new potassium binders warrants prospective cardiovascular outcome trials, such as the ongoing DIAMOND trial (ClinicalTrials.gov Identifier NCT03888066) in HFrEF. Importantly, in the AMBER phase II trial, in 295 patients with resistant hypertension and an eGFR between 25 and $45 \mathrm{~mL} / \mathrm{min} / 1.73 \mathrm{~m}^{2}$ (45\% with heart failure), the potassium binder patiromer, compared to placebo, enabled a more persistent use and greater dose of spironolactone. Two-thirds of the patients in the placebo group developed hyperkalaemia over the 12-week follow-up, with this risk being reduced by half in patients in the patiromer group ${ }^{8}$. The burden of hyperkalaemia associated with steroidal MRA use in patients treated with RAASi could also be alleviated by the use of finerenone ${ }^{9}$ or of other non-steroidal MRAs under development.

Notwithstanding the above, a proper use of RAASi encompasses a proper clinical and laboratory monitoring (namely creatinine and potassium), the latter found to be poor in the heart failure setting after MRA initiation ${ }^{10}$. Walther et al. ${ }^{4}$ also reported that serum potassium concentration was verified within the 6 months prior to discontinuation in only $38.6 \%$ of discontinuation events. Furthermore, while $61 \%$ of patients who discontinued ACEI/ARB restarted within 6 months of discontinuation, less than $50 \%$ of these patients had both creatinine and potassium tested in the first 90 days of ACEI/ARB resumption, which is certainly far from being optimal. The use of RAASi requires frequent $\mathrm{K}$ monitoring or we risk permanent discontinuation of these drugs due to severe hyperkalaemia.

Finally, it should be remembered that the latest therapeutic options shown to decrease residual cardiorenal risk in CKD and/or in HFrEF patients (i.e. SGLT2i in both conditions and the non-steroidal MRA finerenone in diabetic nephropathy ${ }^{9}$ ) were all developed as add-on therapeutics in patients already receiving ACEI or ARBs, thereby encouraging us to avoid therapeutic inertia with these lifesaving drugs in the cardiorenal continuum. Whether RAASi enablement could be -per se- a therapeutic target warrants dedicated prospective trials. 
Keywords: chronic kidney disease- hyperkalaemia - mineralocorticoid receptor antagonist- renin angiotensin aldosterone system inhibitor-cardiorenal syndrome

1. Zannad F and Rossignol P. Cardiorenal Syndrome Revisited. Circulation. 2018;138:929-944.

2. Murphy DP, Drawz PE and Foley RN. Trends in Angiotensin-Converting Enzyme Inhibitor and Angiotensin II Receptor Blocker Use among Those with Impaired Kidney Function in the United States. J Am Soc Nephrol. 2019;30:1314-1321.

3. Pecoits-Filho R, Fliser D, Tu C, Zee J, Bieber B, Wong MMY, Port F, Combe C, Lopes AA, Reichel H, Narita I, Stengel B, Robinson BM, Massy Z and Investigators CK. Prescription of renin-angiotensin-aldosterone system inhibitors (RAASi) and its determinants in patients with advanced CKD under nephrologist care. J Clin Hypertens (Greenwich). 2019;21:9911001.

4. Walther CP, Winkelmayer WC, Richardson PA, Virani SS and Navaneethan SD. Reninangiotensin system blocker discontinuation and adverse outcomes in CKD. Nephrol Dial Transplant. 2021.

5. Epstein M, Reaven NL, Funk SE, McGaughey KJ, Oestreicher N and Knispel J. Evaluation of the treatment gap between clinical guidelines and the utilization of reninangiotensin-aldosterone system inhibitors. Am J Manag Care. 2015;21:s212-20.

6. Rossignol P, Lainscak M, Crespo-Leiro MG, Laroche C, Piepoli MF, Filippatos G, Rosano GMC, Savarese G, Anker SD, Seferovic PM, Ruschitzka F, Coats AJS, Mebazaa A, McDonagh T, Sahuquillo A, Penco M, Maggioni AP, Lund LH and Heart Failure Long-Term Registry Investigators $G$. Unravelling the interplay between hyperkalaemia, reninangiotensin-aldosterone inhibitor use and clinical outcomes. Data from 9222 chronic heart failure patients of the ESC-HFA-EORP Heart Failure Long-Term Registry. Eur J Heart Fail. 2020;22:1378-1389.

7. Evans M, Palaka E, Furuland H, Bennett H, Linde C, Qin L, McEwan P and Bakhai A. The value of maintaining normokalaemia and enabling RAASi therapy in chronic kidney disease. BMC Nephrol. 2019;20:31.

8. Agarwal R, Rossignol P, Romero A, Garza D, Mayo MR, Warren S, Ma J, White WB and Williams B. Patiromer versus placebo to enable spironolactone use in patients with resistant hypertension and chronic kidney disease (AMBER): a phase 2, randomised, double-blind, placebo-controlled trial. Lancet. 2019;394:1540-1550.

9. Bakris G, Agarwal R, Anker SD, Pitt B, Ruilope L, Rossing P, Kolkhof P, Nowack C, Schloemer P, Joseph A and Filippatos G. Effect of Finerenone on Chronic Kidney Disease Outcomes in Type 2 Diabetes. N Engl J Med. 2020.

10. Cooper LB, Hammill BG, Peterson ED, Pitt B, Maciejewski ML, Curtis LH and Hernandez AF. Consistency of Laboratory Monitoring During Initiation of Mineralocorticoid Receptor Antagonist Therapy in Patients With Heart Failure. JAMA. 2015;314:1973-5.

\section{Source of funding : none}


P.R. reports personal fees from Ablative Solutions, AstraZeneca, Bayer, Boehringer-Ingelheim, Corvidia, CVRx, Fresenius, G3P (stocks), Grunenthal, Idorsia, KBP, Novartis, NovoNordisk, Relypsa, Sanofi, Sequana Medical, Servier, Stealth Peptides, Vifor, Vifor Fresenius Medical Care Renal Pharma; and Cofounder: CardioRenal, a company developing sensors for the home monitoring of potassium and creatinine.

R.A. reports personal fees from Akebia, AstraZeneca, Bayer, Boehringer Ingelheim, Diamedica, Eli Lilly, Johnson \& Johnson, Merck, Reata, Relypsa, Inc., a Vifor Pharma Group Company, Sanofi; has served as associate editor of the American Journal of Nephrology, Nephrology Dialysis and

Transplantation; and an author on UpToDate; and received research grants from the US Veterans Administration and the National Institutes of Health 